\title{
Reactive black 5 induced developmental defects via potentiating apoptotic cell death in Zebrafish (Danio rerio) embryos
}

\begin{abstract}
Reactive Black 5 (RB5) dye is one of the most commonly used synthetic reactive dyes in textile, pharmaceutical, food, cosmetics, plastics, photographic and paper industries. RB5 induced dose dependent $\left(1,2.5,5,10,25 \mathrm{mg} \mathrm{L}^{-1}\right)$ exposure and teratogenice alterations on zebrafish embryos were analyzed. The Genotoxicty effect of RB5 on zebrafish was studied at different hours of post fertilization from 12 to $96 \mathrm{hpf}$ such as survival, hatching, heart beat rate and delayed malformation have been studied. However RB5 cause a lethal effect at 48 and $72 \mathrm{hpf}$ on zebrafish embryos. Different concentrations $\left(1,2.5,5,10,25 \mathrm{mg} \mathrm{L}^{-1}\right)$ of RB5 treated with zebrafish larvae have showed significant abnormalities in Tissue ulceration (TU), Tail deformity (TD), Eye defect (E), Yolk sac edema (YSE), Pericardial edema (PE), Bent Spine (BS). The apoptotic inducing of RB5 in the $25 \mathrm{mgL}^{-1}$ concentration were showed severe cell death induced in heart and yolk sac region at 48, 72 and $96 \mathrm{hpf}$. In conclusion, treatments of RB5 at higher concentrations have exhibited lethal impact on zebrafish embryos.
\end{abstract}

Volume 6 Issue 6 - 2018

\author{
Dharmar Manimaran, Ahamed sha Sulthana, \\ Namasivayam Elangovan \\ Department of Biotechnology, School of Bioscience, Periyar \\ University, India
}

\author{
Correspondence: N Elangovan, Department of Biotechnology, \\ School of Bioscience, \\ Periyar University, Salem-6360 I I, India, Tel +919789097|42, Fax \\ 0427-2345 I24, Email elangavannn@gmail.com
}

Received: May 06, 2017 | Published: November 27, 2018

Keywords: abnormalities, embryos, exposure, RB5, zebrafish, tissue

\section{Introduction}

Dyes are estimated that globally, approximately 10,000 tons of synthetic dyes enter in the aquatic ecosystems. ${ }^{1}$ The dyes can adhere to compatible surfaces by solution, by forming covalent bond or complexes with salts or metals, by physical adsorption or by mechanical retention. Reactive dyes (e.g. textile dyes) are known to be highly water-soluble, poly aromatic molecules that are nondegradable and cannot be absorbed well by biological solids. ${ }^{2}$ Textile manufacturing is one of the most polluting industrial sectors because of the release of potentially toxic compounds, such as synthetic dyes, into the environment. Depending on the class of the dyes, their loss in wastewaters can range from $2 \%$ to $50 \%$ of the original dye concentration. Consequently, uncontrolled use of such dyes can negatively affect human health and the ecological balance. ${ }^{3}$ Their effects are on both environmental and human health, synthetic dyestuffs are an important environmental concern. ${ }^{4} \mathrm{RB} 5$ is of intense necessity to remove dyes from wastewater effectively to ensure safe discharge of treated liquid effluent into water bodies. ${ }^{1}$ In the textile industry, up to 200,000 tons of these dyes are lost to effluents every year during the dyeing and finishing operations, due to the inefficiency of the dyeing process..$^{5}$ Recently, the zebrafish has become a prominent vertebrate model for assessing the toxicity of drugs and chemicals. ${ }^{6}$ Therefore, in this study, RB5 shown in Figure 1 induced dependent exposure and teratogenice alterations on zebrafish embryos. The genotoxicty of different hours of post fertilization 12 to $96 \mathrm{hpf}$ such as survival, hatching, heart beat rate and delayed malformation. However RB5 induced zebrafish embryos cause a lethal effect at 48 and $72 \mathrm{hpf}$.

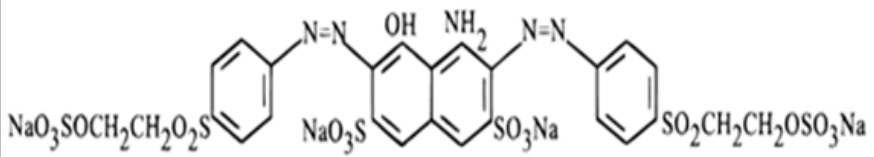

Figure I The structure of reactive black 5 (RB5).

\section{Materials and methods}

\section{Reagents}

The dye Reactive Black 5 (98 \% purity) and Acridine Orange (AO) chemicals were obtained from Sigma Aldrich chemical company (St Louis, MO, USA). All other chemicals used were of analytical grade and highest purity available from commercial sources.

\section{Zebrafish embryo collection and maintenance}

The adult type zebrafish were commercially purchased from local aquarium market. Zebra fish were maintained in a $50 \mathrm{~L}$ glass tank at temperature of $26 \pm 1^{\circ} \mathrm{C}$ with $14: 10 \mathrm{hrs}$ light/dark. Subsequently fed with commercial spirulina micro pelleted food. The zebrafish ratio about 1:2 (female: male) were allocated for breeding in a well meshed chamber. ${ }^{7}$ Subsequently fed with commercial spirulina micro pelleted food. The spawning is triggered once the light is turned on at that time of light source illumination and completed within 30 mins fertilized eggs were collected after spawning and rinsed in distilled water. The fertilized eggs without any abnormalities were taken and maintained in $\mathrm{E} 3$ medium. $^{8}$

\section{Experimental schedule}

The eggs were randomly distributed in to 6 groups in a six well plate $(\mathrm{n}=30$ embryos per well). The embryos in group 1 served as control maintained in E3 medium. The embryos in group 2-6 were exposed with varying concentrations of Reactive Black 5 (RB5) (1, 2.5,5,10 and $25 \mathrm{mg} \mathrm{L}^{-1}$ in E3 medium) for $96 \mathrm{hrs}$ and $50 \%$ of the medium replaced with fresh medium on every $24 \mathrm{hrs}$. The zebrafish embryo development was observed directly under light microscope (Magnus-MLXi, Olympus, Japan) for every $12 \mathrm{hrs} \mathrm{interval.}$

\section{Zebra fish embryo toxicity Assay}

The Reactive Black 5 exposure, we investigated the developmental toxicity profiles such as survival, embryo malformation, hatching and

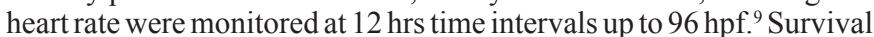
was identified as resting state of heartbeat, failure to develop somite and a non-detached tail. Morphological deformities were examined under a light microscope. The hatched out larva were analyzed for apoptotic analyzed. The development of blastula stage eggs were monitored at above mentioned specified time points after fertilization. Then observation was prolonged until the time of hatching for the 
different exposure groups. Endpoints used for assessing the effects of survival, hatching, malformation and heart beat rates were also noted and described among the juveniles from both control and treated groups were observed under a light microscope.

\section{Apoptotic detection by acridine orange staining}

Apoptosis inducing capacity of Reactive Black 5 was analyzed by acridine orange (AO) staining. ${ }^{10}$ After exposure to Reactive Black 5 for 96 hours post-fertilization, the embryos were washed twice with embryo medium (E3) followed by exposure to $10 \mu$ l acridine orange solution $(5.0 \mu \mathrm{g} / \mathrm{ml}$ in E3 medium) for 20 minutes at room temperature. The embryos were washed with E3 medium and the apoptotic bodies were examined under a fluorescent microscope with an emission range of 525nm (Magnus-MLXi, Olympus Japan).

\section{Statistical analysis}

Data were analyzed using the statistical package Graphad Prism version 5.0. The $\mathrm{IC}_{50}$ values were calculated. Data for toxicity tests RB5 values are expressed as mean \pm SEM in three independent experiments. A One way and two way ANOVA of Dunnets multiple compare the differences between treatment and control groups. Differences were considered statistically significant $p<0.05$.

\section{Results}

\section{RB5 modulates survival rate on zebrafish embryo}

The survival of zebrafish embryos after exposed to different concentration of RB5 was determined at specific time intervals (12, $24,36,48,60,72,84$, and $96 \mathrm{hpf}$ ) was shown in Figure 2. Exposure to RB5 at lower concentrations $\left(<5 \mathrm{mg} \mathrm{L}^{-1}\right)$ caused mild alterations in survival rate whereas, embryos exposed with higher concentrations $\left(>5 \mathrm{~m} \mathrm{~L} \mathrm{~L}^{-1}\right)$ showed drastic reduction in survival rate when compared to control. Since, the variations in survival rate was not observed in embryos maintained in medium alone. The concentration required to inhibit $50 \%\left(\mathrm{IC}_{50}\right)$ embryo survival was achieved at $0.5171 \mathrm{mgL}$ ${ }^{1}$. These results depict the direct interference of RB5 on zebrafish embryo by modulating the normal survival rate.

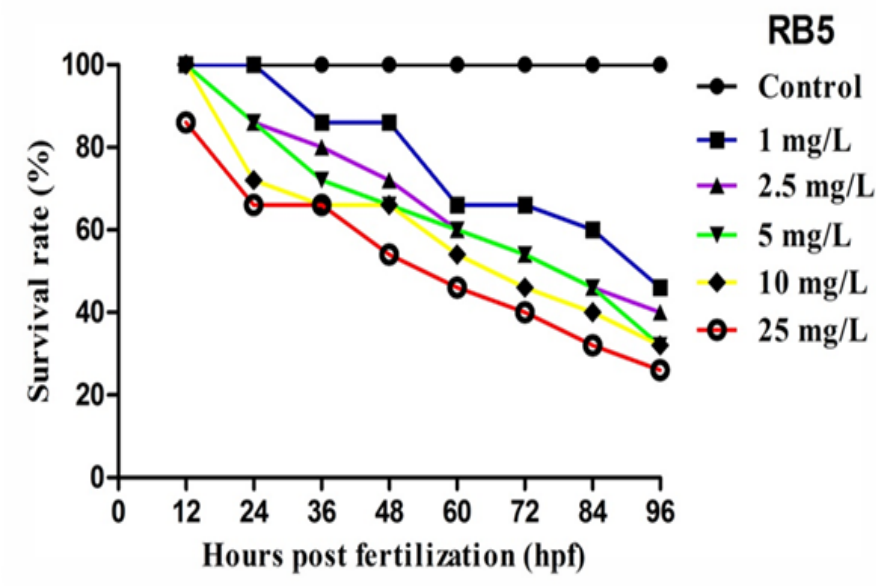

Figure 2 Effect of RB5 on survival rate of zebrafish embryos over 96hpf. Values are expressed as mean $\pm \operatorname{SEM}(n=3)$.

\section{Delayed hatching rate of RB5 on zebrafish embryo}

Hatching success of all control and RB5 post-treated zebrafish embryos were illustrated in Figure 3. The RB5 treated embryos showed a dose dependent delayed hatching rate when compared to the control group. RB5 treated zebrafish embryo at higher concentration such as 10 and $25 \mathrm{mg} \mathrm{L}^{-1}$ showed significantly $(\mathrm{P}<0.05)$ delayed in hatching success whereas, embryos maintained in medium alone not showed any defective variations.

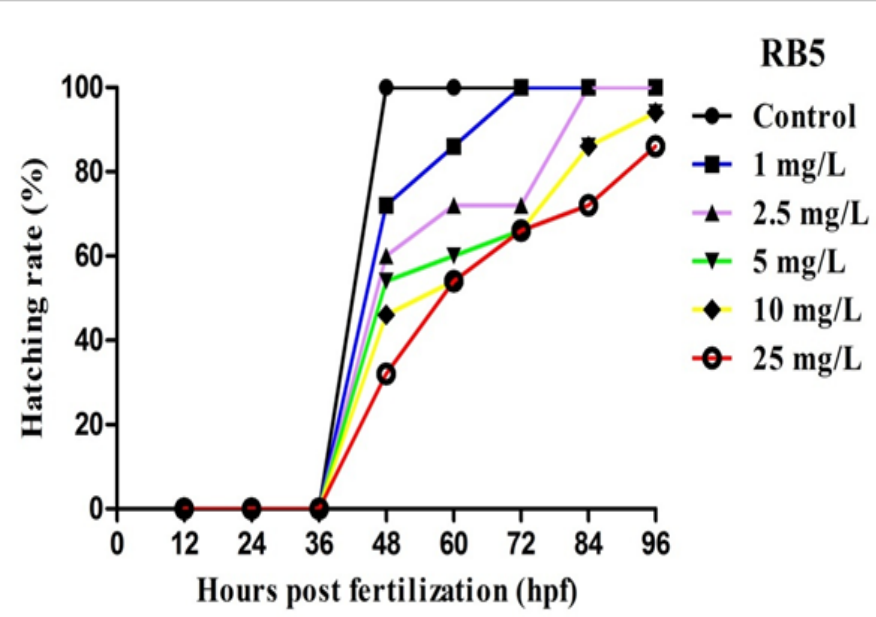

Figure 3 Graph represents the hatching rate of zebrafish embryos exposed to RB5 for $96 \mathrm{~h}$. Values are expressed as mean $\pm \operatorname{SEM}(n=3)$.

\section{Effects of RB5 on heart beat rate}

Cardiac function hindrance capacity of RB5 dye stuff on all experimental zebrafish embryos at specific time intervals (48 and $84 \mathrm{hpf}$ ) was shown in Figure 4. Control embryos exhibits normal functioning of heart beat. Embryos exposed with lower concentration of RB5 $(1,2.5 \mathrm{mg} / \mathrm{L})$ showed no variations in heart rate whereas, reactive dye exposed with higher concentration significantly $(\mathrm{P}<0.05)$ induces reduced heart rate when compared to control group.

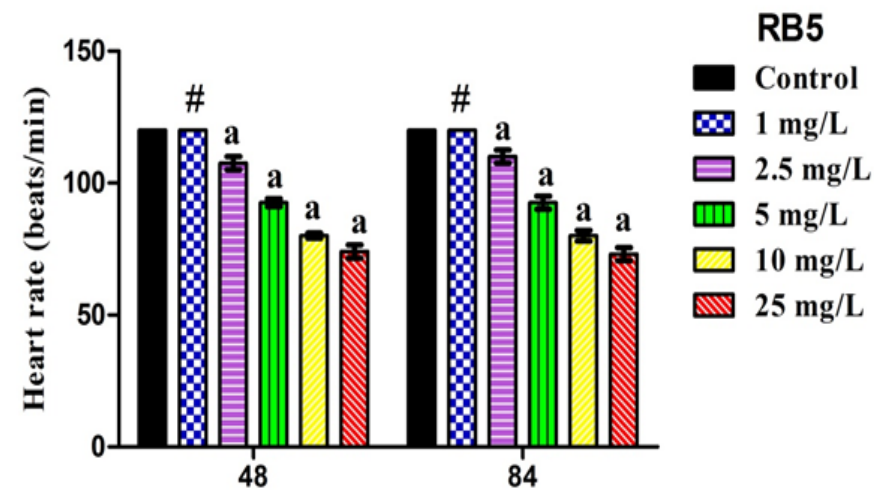

Hours post fertilization (hpf)

Figure 4 Graphical representation elucidates the measurement of heart beat rate after exposed to RB5 on zebrafish embryos at specific time intervals 48 and $84 \mathrm{hpf}$. Values are expressed as mean \pm SEM $(n=3)$ and the values are statistically significant at $\mathrm{P}<0.05$ by dunnett's test. Comparisons $\mathrm{aP}<0.05$ vs control; \#non-significant vs control group.

\section{Effect of RB5 on phenotypic alterations}

The developmental deformities induced by RB5 at dosedependent levels on zebrafish embryos as shown in Figure 5. Control embryos showed normal appearance of morphological development without any sign of abnormalities. The embryos treated with lower concentrations of RB5 $\left(1,2.5 \& 5.0 \mathrm{mg} \mathrm{L}^{-1}\right)$ showed mild alterations such as Tissue ulceration (TU), Tail deformity (TD), Eye defect (E), Yolk sac edema (YSE), Pericardial edema (PE), Bent Spine (BS). However, higher concentration of RB5 $\left(>10 \mathrm{mg} \mathrm{L}^{-1}\right)$ showed highly reproducible pattern of above mentioned deformities, when compared to control. This result suggests that multiple or targeting capacity of RB5 dye on zebrafish embryos affected more severe and higher rates of deformity. 


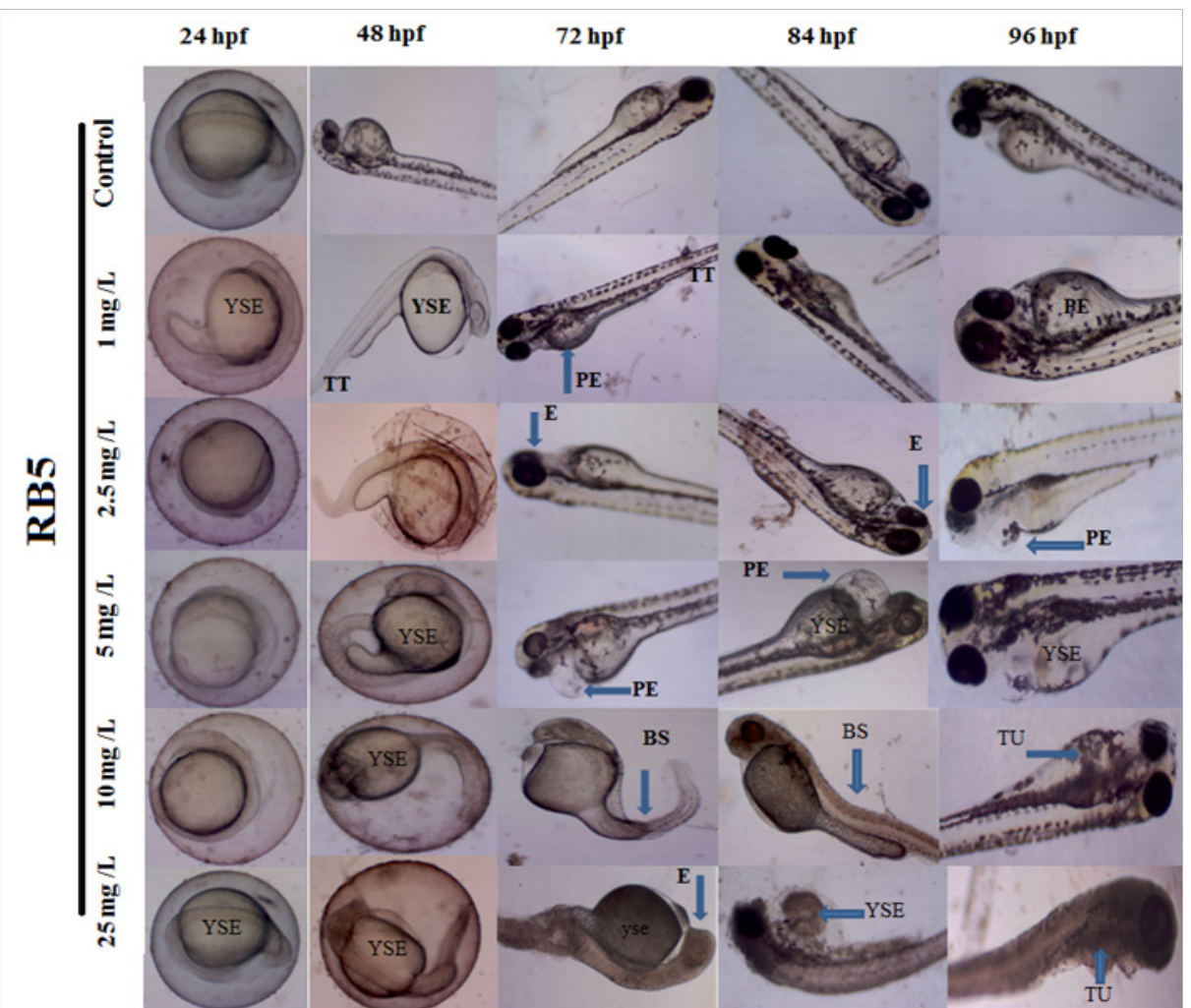

Figure 5 Photomicrograph representing the developmental deformities induced by RB5 on zebrafish embryos between 24 to 96 hpf. Blue arrow show tissue ulceration (TU), Tail deformity (TD), Eye defect (E), Yolk sac edema (YSE), Pericardial edema (PE) and Bent Spine (BS) were seen (Magnification 4x).

\section{RB5 induces apoptotic patterns on zebrafish larvae}

The apoptotic inducing capacity of RB5 on all experimental embryos after stained with acridine orange (AO) was shown in the figure 6. No appearance of apoptotic cells was observed in control larvae at 96 hpf. RB5 treated cells at 5, 10 and $25 \mathrm{mg} \mathrm{L}^{-1}$ showed a considerable number of apoptotic cells as shown in green fluorescent, mainly around in yolk sac and heart regions, which represent its apoptotic inducing ability of RB5 after 96 hrs. The presence of bright green region indicates the presence of apoptotic cell death.

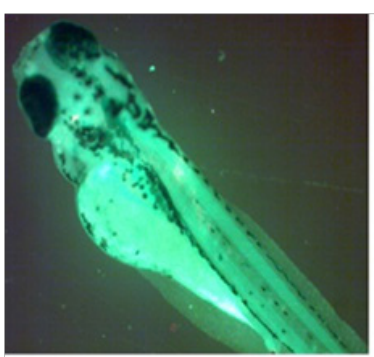

Control

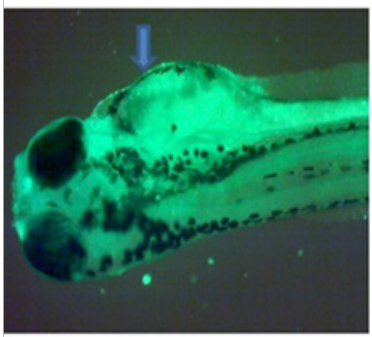

$5 \mathrm{mg}$

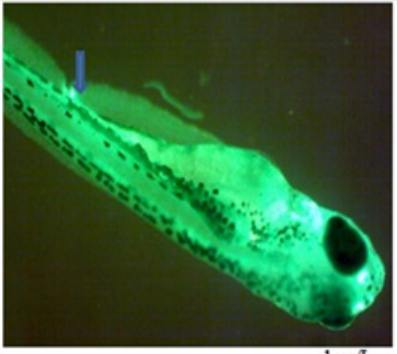

$1 \mathrm{mg} \mathrm{L}$

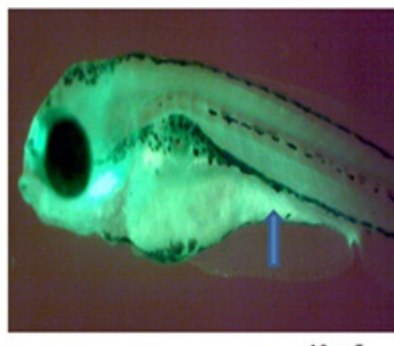

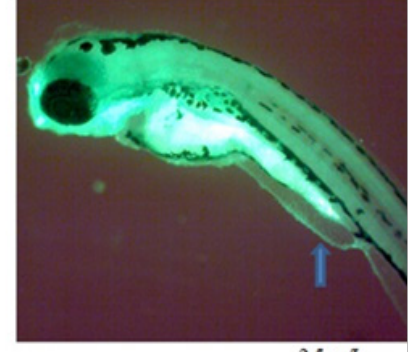

$2.5 \mathrm{mg} \mathrm{L}$

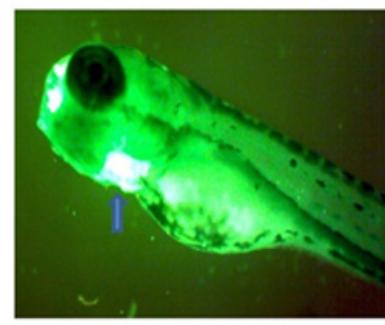

$25 \mathrm{mg} \mathrm{L}$

Figure 6 Apoptotic inducing capacity of RB5. Apoptotic cells stained with AO appeared mainly in the tail and body region. Apoptotic cells and abnormalities are indicated by arrows. 


\section{Discussion}

The potential hazards of textile effluent to ecosystems and human health have aroused great concern since textile effluents usually contain some toxic substances, such as additives, detergents, surfactants and dyes, which are carcinogenic, mutagenic or teratogenic to various organisms. ${ }^{11}$ RB5 is a commercial azo dye used for dyeing of cotton fibers and are the most important class of synthetic organic dyes but the toxicological database of RB5 is not yet proven. This study investigated the effects of Reactive Black 5 (RB5) exposure on early developing zebrafish embryos. It was revealed that exposure to low doses of RB5 $\left(\leq 5 \mathrm{mgL}^{-1}\right)$ did not significantly affect the embryos' survival or cause obvious malformation during the embryonic stages (96 hpf). However, exposure to higher concentrations of arsenate $\left(\geq 5 \mathrm{mg} \mathrm{L}^{-1}\right.$ ) reduced survival and caused changes in embryonic development, including delayed hatching, reduced growth and impairments in heart beat function. ${ }^{12}$ In this study, we noticed significantly declined hatching rates upon exposure to RB5, which might have been due to alterations in the cleavage enzymes produced by hatching glands in chorionic region.

The retardation in hatching rate might be due to disturbance of the hatching enzyme that is produced by a hatching gland embedded in the chorionic sac resulting in the shortage of oxygen supply essential to the development of embryos. Several lines of evidence addressing the same line of mechanism behind hatching failure. ${ }^{13,14}$ It has been documented that equilibrium between cell proliferation and apoptosis is a key factor in embryogenesis, and it can reflect the dynamic aspects of the development of the neural tube and lengthening of the trunk and tail..$^{15}$ In this study, we found that the RB5 treated embryos displayed abnormal cell proliferation and apoptosis in the heart and yolk sac regions, where the cardiac effects were verified. This suggests that the abnormal cell proliferation and apoptotic bodies may partly account for the heartbeat defects. Several reports stated that exposure to synthetic textile dyes potentiated DNA damage and inhibit DNA repair by generation of reactive oxygen species (ROS) and inhibition of DNA ligase. ${ }^{16}$ Although the concentration of RB5 that we determined to be toxic is greater than that commonly reported in the environment, we believe our results are of importance and merit further study because of the increased use of RB5, and its increasing frequency of detection in the environment, making it a potentially significant source of future toxicity in other vertebrates.

\section{Conclusion}

In conclusion the present study shows that RB5 could effectively cause severe abnormalities on zebrafish embryos at the concentration above $10 \mathrm{mg} \mathrm{L}^{-1}$ as it was evidenced by severe abnormalities, altered survival, hatching success, and Heart beat rate. This study revealed the induction of toxicity by the textile dye Reactive Black 5 to the zebrafish embryos. Consequently, dyes have to be removed in dye wastewater before discharge because of its toxic efficacy.

\section{Acknowledgments}

We gratefully acknowledge our colleagues in the zebrafish field for their collective contributions, with special thanks to all members of Elangovan Lab.

\section{Conflicts of interest}

Authors declare that they have no conflict of interest concerning this article.

\section{References}

1. Ferraz ER, Grando MD, Oliveira DP. The azo dye Disperse Orange 1 induces DNA damage and cytotoxic effects but does not cause ecotoxic effects in Daphnia similis and Vibrio fischeri. J Hazard Mater. 2011;192(2):628-633.

2. Hu YL, Qi W, Han F, et al. Toxicity evaluation of biodegradable chitosan nanoparticles using a zebrafish embryo model. Int J Nanomedicine. 2011;4:3351-3359.

3. De Oliveira GA, de Lapuente J, Teixidó E, et al. Textile dyes induce toxicity on zebrafish early life stages. Environ Toxic Chem. 2016;35(2):429-434.

4. Oliveira GA, Ferraz ER, Chequer FM, et al. Chlorination treatment of aqueous samples reduces, but does not eliminate, the mutagenic effect of the azo dyes Disperse Red 1, Disperse Red 13 and Disperse Orange 1. Mutat Res. 2010;703(2):200-208.

5. Ogugbue CJ, Sawidis T. Bioremediation and Detoxification of Synthetic Wastewater Containing Triarylmethane Dyes by Aeromonas hydrophila Isolated from Industrial Effluent. Biotechnol Res Int. 2011;967925:1-11.

6. Rajaguru P, Fairbairn LJ, Ashby J, et al. Genotoxicity studies on the azo dye Direct Red 2 using the in vivo mouse bone marrow micronucleus test. Mutat Res. 1999;444(1):175-180.

7. Berry JP, Gantar M, Gibbs PD, et al .The zebra fish (Danio rerio) embryo as a model system identification and characterization of developmental toxins from marine and freshwater microalgae. Comp Biochem Physiol C Toxicol Pharmacol. 2007;145(1):61-72.

8. Brant M, Granato M, Nusslein VC, et al. Zebra fish a practical approach. Oxford university press; 2002. 7-37 p.

9. Kimmel CB, Ballard WW, Kimmel SR, et al. Stages of embryonic development of the zebra fish. Dev Dyn. 1995;203(3):253-310.

10. Chan P, Cheng S. Cadmium-induced ectopic apoptosis in zebra fish embryos. Arch Toxicol. 2003;77(2):69-79.

11. Sumathi MK, Kalaiselvi K, Palanivel M, et al. Genotoxicity of textile dye effluent on fish (Cyprinus carpio) measured using the comet assay. Bull Environ Contam Toxicol. 2001;66(3):407-414.

12. Cheng JP, Flahaut E, Cheng SH. Effect of carbon nanotubes on developing zebrafish (Danio rerio) embryos. Environ Toxicol Chem. 2007;26(4):708-716.

13. Xia TA, Zhao Y, Sager T, et al. Decreased dissolution of $\mathrm{ZnO}$ by iron doping yields nanoparticles with reduced toxicity in the rodent lung and zebrafish embryos. ACS Nano. 2011;5(2):1223-1235.

14. Wullimann MF, Knipp S. Proliferation pattern changes in the zebrafish brain from embryonic through early post embryonic stages. Anat Embryol (Berl). 2000;202(5):385-400.

15. Jang GH, Park IS, Lee SH, et al. Malachite green induces cardiovascular defects in developing zebrafish (Danio rerio) embryos by blocking VEGFR-2 signaling. Biochem Biophys Res Commun. 2009;382(3):486-491.

16. Liu F, Gentles A, Theodorakis CW. Arsenate and perchlorate toxicity,growth effects, and thyroid histopathology in hypothyroid zebrafish Danio rerio. Chemosphere. 2007;71(7):1369-1379. 